\title{
Evaluation of ZigBee Topology Effect on Throughput and End to End Delay Due to Different Transmission Bands for IoT Applications
}

\author{
Yehia R. Hamdy, and Ahmed I. Alghannam
}

\begin{abstract}
ZigBee is widely used in wireless network in Internet of Things (IoT) applications to remotely sensing and automation due to its unique characteristics compared to other wireless networks. According to ZigBee classification of IEEE 802.15.4 standard, the network consists of four layers. The ZigBee topology is represented in second layer. Furthermore, the ZigBee topology consists of three topologies, star, tree and mesh. Also there are many transmission bands allowed in physical layer, such as $2.4 \mathrm{GHz}, 915 \mathrm{MHz}, 868 \mathrm{MHz}$. The aim of this paper is to evaluate the effect of ZigBee topologies on End to End delay and throughput for different transmission bands. Riverbed Modeler is used to simulate multiple ZigBee proposed scenarios and collect the results. The results of the study recommend which topology should be used at each transmission band to provide lowest End to End delay or obtain maximum throughput, which is case sensitive in some IoT applications that required for example minimum delay time or sending high amount of data.
\end{abstract}

Keywords-component, ZigBee Topology, Throughput, End to End Delay, Internet of Things (IoT).

\section{INTRODUCTION}

$\mathrm{Z}$ $\mathrm{gBee}$ is a wireless network specified by IEEE 802.15.4 standard, it is mainly used in Internet of Things (IoT) and automation such as factories, plants to keep monitoring of production lines. It can be also used in hospitals and medical facilities for data collections of medical devices [1]. Other usage is at homes, offices and any other places that need to be monitored, automated or when a data needed to be collected [2].

The characteristics of ZigBee network make it appealing to be used for remotely sensing instead using other wireless networks. These characteristics are: low-power consumption, ability to support large number of devices, and considerably small time-delay. In a typical ZigBee network, the end devices or sensors which are called nodes can operate for several days with single charge due to fact its low data rate transition and the end devices can be put to sleep when it's not used. The number of end devices which can be supported by the single coordinator or control center is around 65000 end device

Manuscript received December 15, 2019; revised April 5, 2020. Date of publication July 20, 2020. Date of current version July 20, 2020.

Authors are with the Electrical Engineering Department, College of Engineering, University of Mosul, Mosul, Iraq (e-mails: \{yehia.rehab, ahmed_edrees\}@uomosul.edu.iq).

Digital Object Identifier (DOI): 10.24138/jcomss.v16i3.975 depending on the type of topology used [3]. The coverage area of ZigBee network can be up to 100 meters specified by IEEE 802.15.4 standard, the communication in this range translated to considerably small time delay.

The most important factors in every wireless network are the delay and throughput, and since ZigBee is a wireless network, these two factors must be taken in consideration whenever ZigBee is implemented. The delay and throughput in ZigBee are largely affected by the type of topology and the transmission band used.

When ZigBee is applied in IoT specific application, the ZigBee topology must be chosen according to available transmission band to satisfy delay or throughput for that specific IoT application. For example, if ZigBee is used to monitor patient in emergency room of a hospital, the doctors must know if his heart or lungs stop working as soon as possible, which required minimum delay, so the planner must choose the topology that satisfy minimum delay at the transmission band available, because this IoT application is sensitive to time. Other IoT applications might require higher throughput (collect large number of data) but the delay is not that critical. For example, when ZigBee is used in IoT application that monitor protected agriculture farm for humidity, temperature, sunlight exposure, ... etc. In this case the planner must know which ZigBee topology should be used among the three topologies at the transmission band available to achieve highest throughput, because this IoT application required to collect large amount of data, but the delay is not that sensitive. The goal of this paper is to evaluate these parameters so ZigBee planners can optimize the network for specific use in IoT applications.

The rest of this paper will be organized as follows; Section II reviews the related work. Section III presents ZigBee IEEE 802.15.4 network standard. Section IV presents an overview of ZigBee topologies. Section V presents the proposed simulation scenario. Section VI presents the results obtained from proposed simulation. Finally, section VII concludes the results and recommends the use of topology according to throughput and delay required by the IoT application.

\section{RELATED WORKS}

This section briefly reviews the research related to topology and transmission bands of ZigBee networks. In [4], the authors investigate the star and mesh topology metrics such as throughput, load and MAC delay for three different industrial, scientific and medical radio bands: $868 \mathrm{MHz}$ (EU and Japan), 
$915 \mathrm{MHz}$ (ISM, US) and $2.4 \mathrm{GHz}$ (Worldwide). The tree topology is not investigated in this paper also the end to end delay is not investigated, the study conducted using OPNET simulator. In [5], the authors analysis the performance of ZigBee tree and mesh topologies by using different frequency bands. The performance of throughput, queue size, data dropped, MAC delay and hop number are analyzed using OPNET Modeler 14.5 simulator. The paper did not study star topology or the end to end delay. In [6], the authors investigate the effect of transmission power on throughput, MAC load, MAC delay and end to end delay for tree and mesh topologies. The paper tries to optimize the performance of tree and mesh topologies according to transmitted power. The simulation conducted using OPNET. In [7], the authors analysis the performance of star, tree and mesh topologies for increased number of nodes. The paper study the effect of increasing the number of nodes (sensors) on end to end delay, throughput and number of hops for star, tree and mesh topologies. The study use Riverbed Modeler for simulation.

\section{ZIGBEE IEEE 802.15.4 NETWORK}

The general specification of IEEE 802.15.4 ZigBee wireless network can be summarized in table I.

TABLE I

GENERAL SPECIFICATION OF IEEE 802.15.4 STANDARD

\begin{tabular}{|c|c|}
\hline Parameter & Value \\
\hline Transmission Range (meters) & $\leq 100$ (Non-Line of Sight) \\
\hline Throughput (Kb/s) & $\begin{array}{c}\text { 20-250 (Depending on } \\
\text { Topology) }\end{array}$ \\
\hline $\begin{array}{c}\text { Network Size (No. of Nodes } \\
\text { Supported by Single } \\
\text { Coordinator) } \\
\end{array}$ & $\begin{array}{c}\leq 65000 \text { (Depending on } \\
\text { Topology) }\end{array}$ \\
\hline $\begin{array}{c}\text { Battery Life of Sensors (Days } \\
\text { per One Charge) }\end{array}$ & $\begin{array}{c}\leq 1000 \text { (Depending on } \\
\text { Sensor })\end{array}$ \\
\hline
\end{tabular}

According to the specifications of IEEE 802.15.4, ZigBee networks can be classified into four layers as in table II.

The upper two layers of ZigBee Networks which are application layer, Network and security layers specified by ZigBee Alliance to provide manufacturing standards [8]. The lower two layers, the MAC layer and Physical Layer are specified by IEEE 802.15.4 standard to ensure not to interfere with other wireless protocols.

TABLE II

LAYERS CLASSIFICATION OF ZIGBEE NETWORK

\begin{tabular}{|c|}
\hline Application Layer \\
\hline Network and Security Layer \\
\hline Medium Access Control (MAC) Layer \\
\hline Physical Layer \\
\hline
\end{tabular}

To understand the operation of ZigBee network the components of the network must be understood clearly. ZigBee network consist of three components: ZigBee coordinator, ZigBee router, and ZigBee end device or sensor.

ZigBee coordinator is the core node of the network. Its responsible for initializing the network, selecting appropriate channel, and connecting other devices like routers and end devices in the network. In any ZigBee network there can be only one coordinator.

ZigBee router is a mid-device which can be used to connect between ZigBee coordinator and ZigBee end devices. The use of the router depends on the type of topology used. Its main function is routing traffic between coordinator and end devices.

The final element of ZigBee network which is the end device or the sensor. Its function is collecting data which are been automated or monitored. It can be connected directly to the coordinator or to a ZigBee router depending on the type of topology used [9].

\section{ZIGBEE TOPOLOGY}

The topology of ZigBee network is the set of configuration that consists of: coordinator, routers and end devices which form the network depending on the type of connection between these three kind of nodes. The configuration of ZigBee topology also consists of different protocols and routing algorithms that used for routing traffic inside the ZigBee network. The network and security layer is responsible for determining the type of topology for ZigBee Network [10]. There are three type of topology: Star Topology, Tree Topology and Mesh Topology.

\section{A. Star Topology}

This topology is the simplest and the most limited among the other types of topologies in ZigBee networks. In this topology the configuration of the network consists of coordinator which is located at the centre of the network and end devices which are distributed in the network. All end devices are connected directly to the coordinator and all the traffic routing between end devices is happened through the coordinator. Thus using routers is not logical as they only can work as end devices and cannot be used for routing the traffic. The advantage of this topology is the simplicity of configuration [11]. The disadvantages are that there is no alternative routing for data traffic if the connection between the end device and coordinator is lost. Also the coordinator can be congested with the large amount of packets since all end devices are connected directly to the coordinator.

\section{B. Tree Topology}

In this topology the coordinator is connected to several routers and end devices. The routers can also be connected to other routers and end devices, and this can continue to a certain number of levels. The hierarchy structure of this topology is like a tree with the coordinator at the top of it. In this topology there can only be one coordinator and a number of routers and end devices [12]. The coordinator and routers are considered as branches of this tree, therefore can have leaves which are the end devices. On the other hand, the leaves or end devices cannot have another leaves, or cannot be connected to other end devices. In this topology the transmission between end devices and coordinator are take place by routers [11]. Thus, when two end devices want to communicate, the transmission go through router to coordinator, then from coordinator to router to end device and vice versa. The disadvantage of this topology is that there is no alternative route if the connection to reach destination fails. 


\section{Mesh Topology}

This Topology has a structure similar to that of Tree Topology from the build perspective, but with more flexibility in transmission side. There is also one coordinator in this topology which is connected to several routers. The routers can be connected to other routers or end devices (sensors), but the end devices cannot be connected to other end devices. The transmission goes from end device to routers and then to the coordinator. However, the routers can communicate with other routers or end devices directly without the connection goes through the coordinator [11]. Also the end devices can communicate with other routers or end devices without the need the transmission to go through the coordinator. Thus the transmission is more flexible and can be done by directing algorithm.

\section{Proposed Simulation ScEnarios}

The Riverbed Modeler Academic Edition 17.5 is used to simulate the ZigBee network and obtain results. To study the effects of ZigBee topology on throughput and end to end delay for different transmission bands, three scenarios have been created in Riverbed Modeler simulation program depending on the assumptions in the table III.

TABLE III

SIMULATION PARAMETERS

\begin{tabular}{|c|c|c|c|}
\hline & \multicolumn{3}{|c|}{ Zigbee Topology } \\
\hline & Star & Tree & Mesh \\
\hline No. Coordinators & 1 & 1 & 1 \\
\hline No. End Devices & 18 & 18 & 18 \\
\hline No. Routers & 0 & 8 & 8 \\
\hline $\begin{array}{c}\text { Transmission } \\
\text { bands }\end{array}$ & $\begin{array}{l}2.4 \mathrm{GHz} \\
915 \mathrm{MHz}, \\
868 \mathrm{MHz}\end{array}$ & $\begin{array}{c}2.4 \mathrm{GHz}, \\
915 \mathrm{MHz}, \\
868 \mathrm{MHz}\end{array}$ & $\begin{array}{c}2.4 \mathrm{GHz}, \\
915 \mathrm{MHz}, \\
868 \mathrm{MHz}\end{array}$ \\
\hline Transmit power & $0.05 \mathrm{w}$ & $0.05 \mathrm{w}$ & $0.05 \mathrm{w}$ \\
\hline Data rates & $\begin{array}{c}250 \mathrm{KBPS}, \\
40 \mathrm{KBPS}, \\
20 \mathrm{KBPS}\end{array}$ & $\begin{array}{c}250 \mathrm{KBPS}, \\
40 \mathrm{KBPS}, \\
20 \mathrm{KBPS}\end{array}$ & $\begin{array}{c}250 \mathrm{KBPS}, \\
40 \mathrm{KBPS}, \\
20 \mathrm{KBPS}\end{array}$ \\
\hline
\end{tabular}

The scenarios simulate the three ZigBee topologies; star, tree and mesh described in the section above. The area of ZigBee network is taken $100 \times 100$ meter for the three scenarios. To evaluate the effect of ZigBee topology performance regarding throughput and end to end delay, the three transmission band specified for ZigBee standard are compared.

For the three scenarios used in simulation, the number of coordinators used is one in the middle of simulation area. The number of end devices or sensors are set to 18 end device, and the number of routers used set to 8 routers except for the star topology scenario since there is no rule for routers in directing of data. The transmitted power is kept constant at 0.05 watt at all scenarios. Three transmission bands of ZigBee Network which are: $2.4 \mathrm{GHz}, 915 \mathrm{MHz}$, and $868 \mathrm{MHz}$ are studied. For each transmission band the three scenarios are applied and evaluate the performance regarding the throughput and end to end delay. The network topologies (Star, Tree and Mesh) are shown in Fig. 1 (a), (b), (c) respectively.

\section{RESULTS}

The results of simulation are divided into three categories depending on the transmission bands used in this study, which are $2.4 \mathrm{GHz}, 915 \mathrm{MHz}$ and $868 \mathrm{MHz}$.

\section{a) $2.4 \mathrm{GHz}$ transmission band}

For the transmission band of $2.4 \mathrm{GHz}$, three scenarios are built according to table 3 parameters. The results of the three ZigBee topologies: star, mesh and tree are shown in Fig. 2 and Fig. 3 regarding throughput and end to end delay respectively.

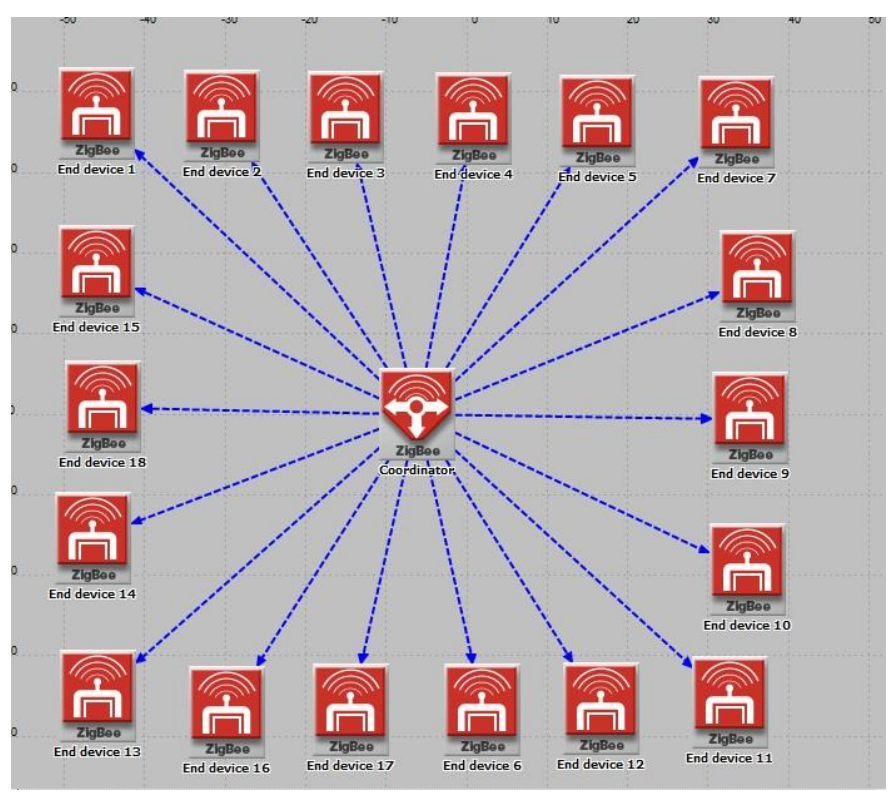

(a)

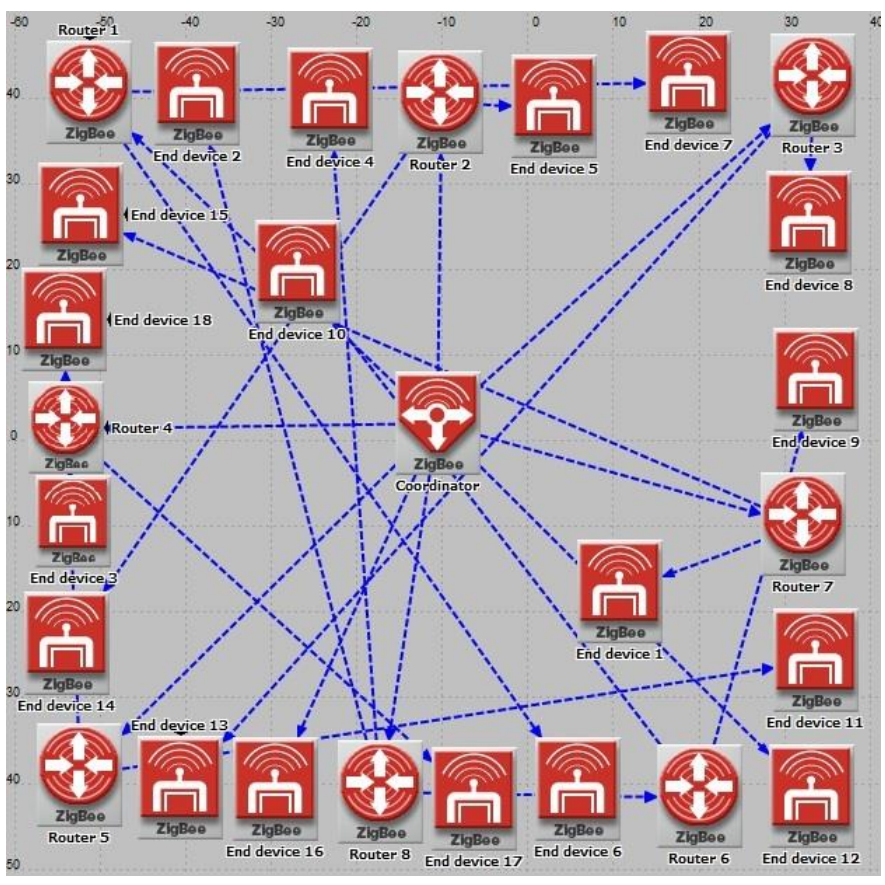

(b) 


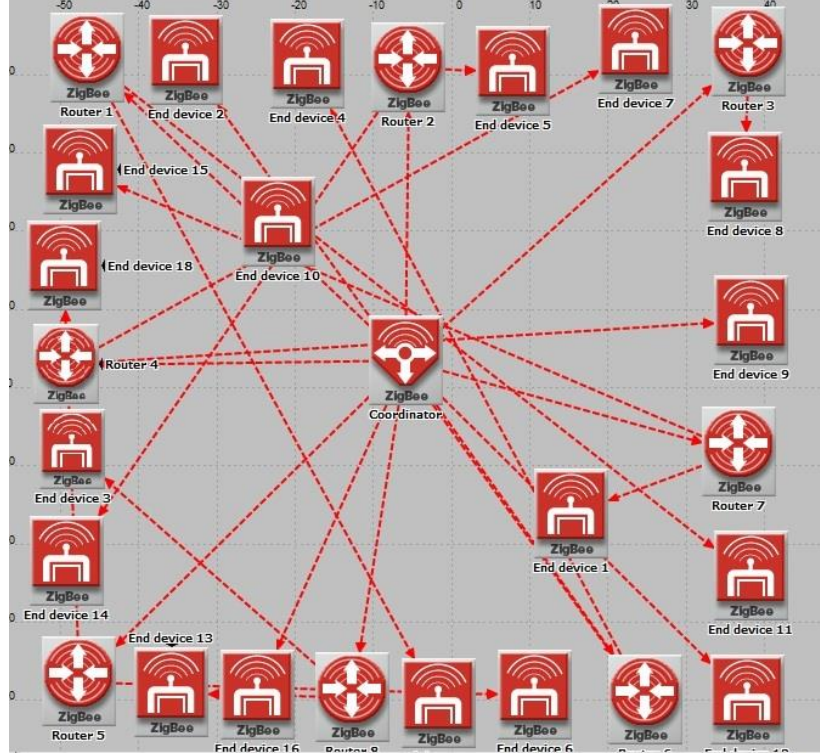

(c)

Fig. 1. (a) Star Topology (b) Tree Topology (c) Mesh Topology "screenshots from the simulator"

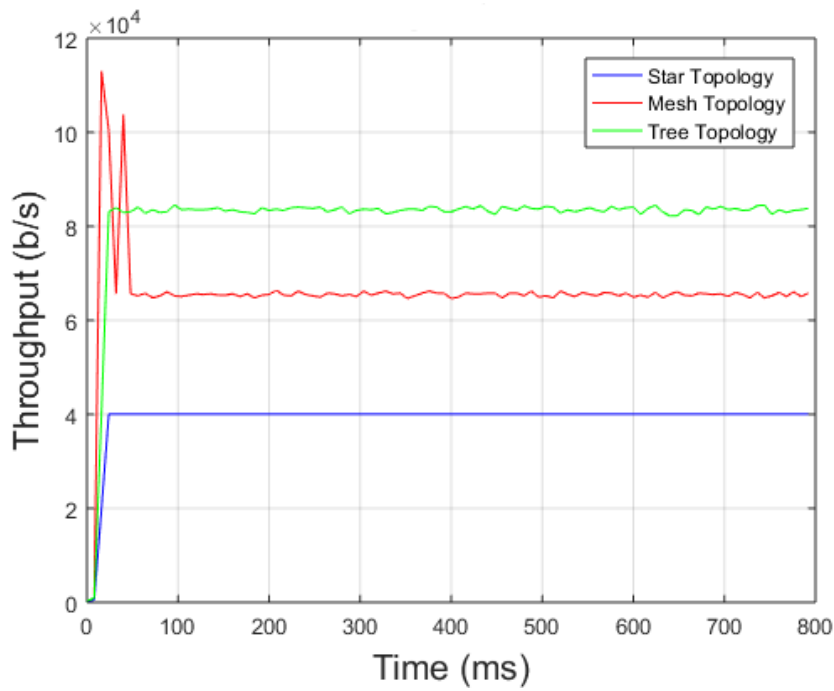

Fig. 2. Throughput (bit per sec) of the three ZigBee topologies at $2.4 \mathrm{GHz}$ transmission band.

From Fig. 2 its found that the tree topology has the best throughput at $2.4 \mathrm{GHz}$ transmission band flowed by mesh and star. While the largest end to end delay for $2.4 \mathrm{GHz}$ from Fig. 3 is for tree topology followed by mesh and star.

\section{b) $915 \mathrm{MHz}$ transmission band}

For this transmission band, the same simulation scenarios used from Fig. 1 and table III. The results of simulation for throughput is shown in Fig. 4 and end to end delay in Fig. 5. For the three ZigBee topologies. The throughput of star topology from Fig 4 is found to be the best for $915 \mathrm{MHz}$ transmission band.

The throughput of mesh and tree topologies are convergent with better throughput of mesh topology. From Fig. 5 the largest end to end delay is for star topology while the best one is for this transmission band is tree topology.

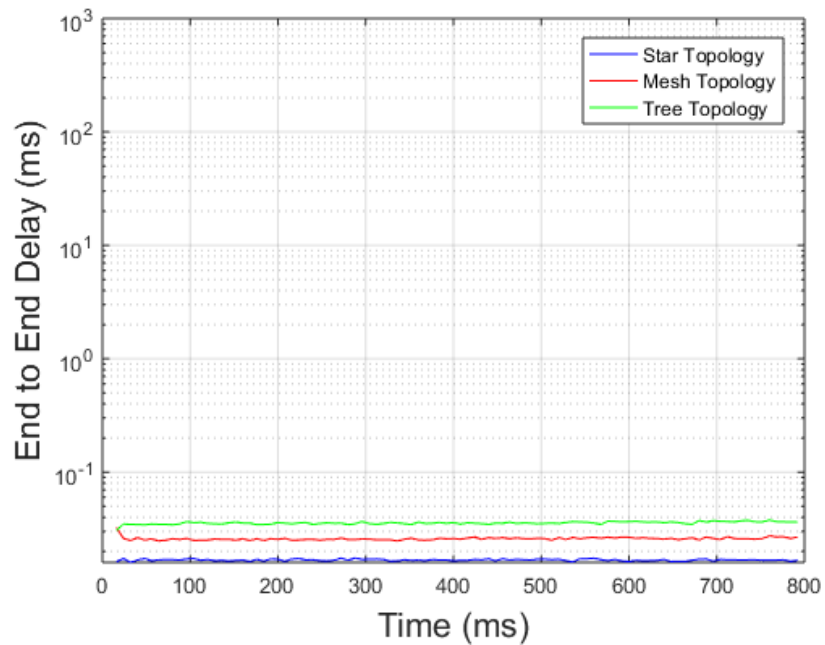

Fig. 3. End to End Delay (mili sec) of the three ZigBee topologies at $2.4 \mathrm{GHz}$ transmission band.

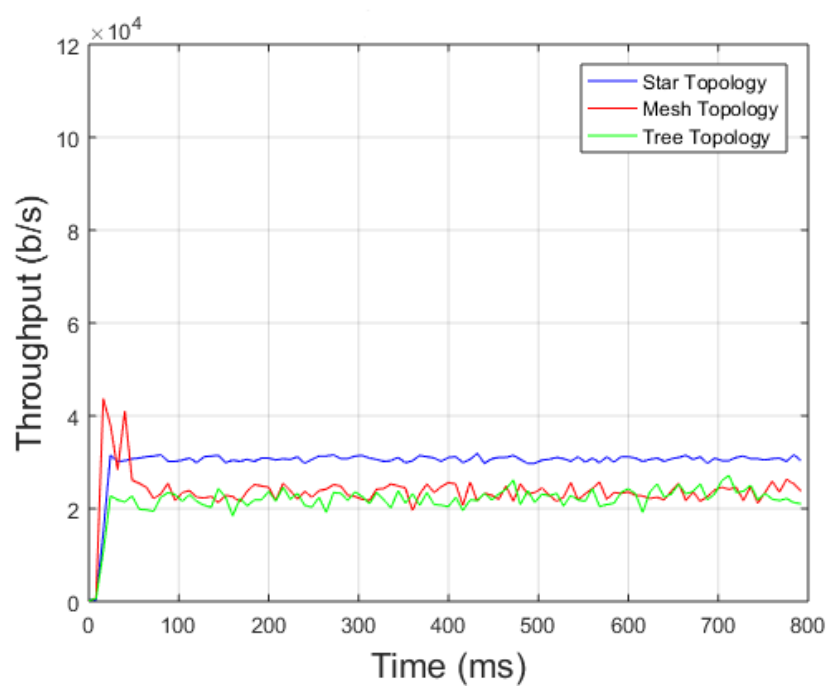

Fig. 4. Throughput (bit per sec) of the three ZigBee topologies at $915 \mathrm{MHz}$ transmission band.

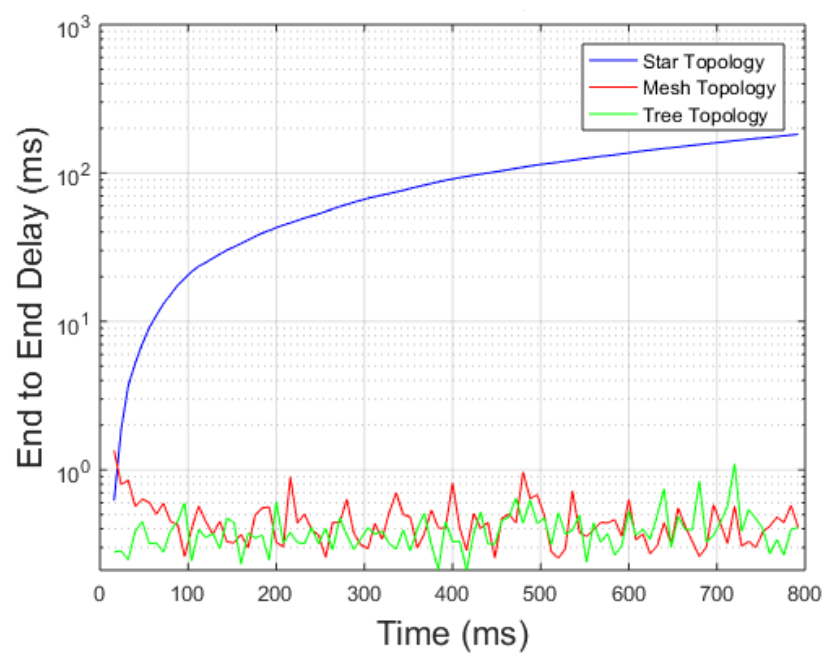

Fig. 5. End to End Delay (mili sec) of the three ZigBee topologies at 915 $\mathrm{MHz}$ transmission band. 


\section{c) $868 \mathrm{MHz}$ transmission band}

The results of simulation for the lest transmission band of ZigBee network at $868 \mathrm{MHz}$ are shown in Fig. 6 and Fig. 7 for throughput and end to end delay respectively.

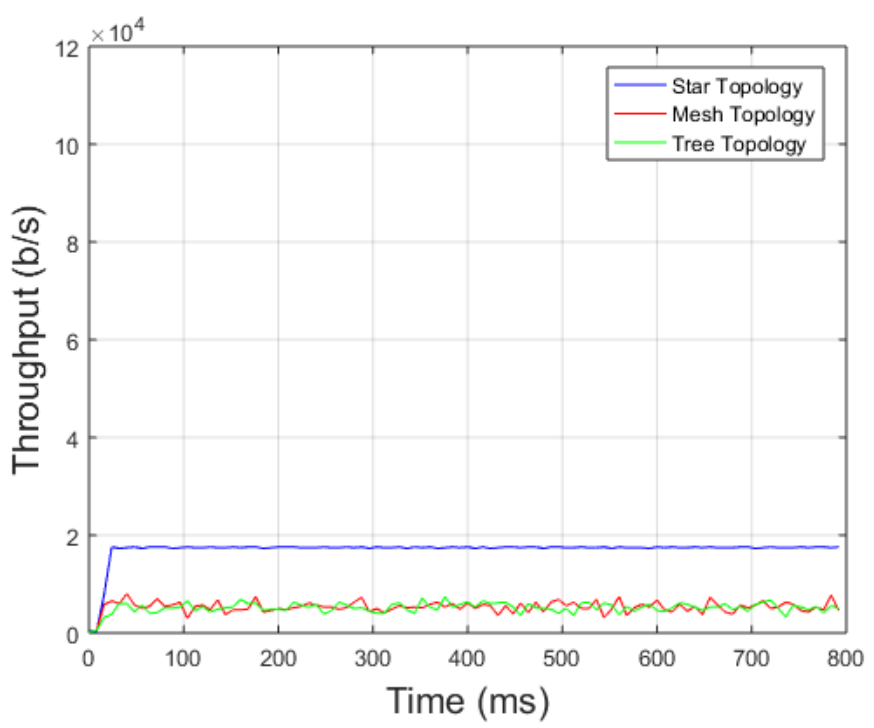

Fig. 6. Throughput (bit per sec) of the three ZigBee topologies at $868 \mathrm{MHz}$ transmission band.

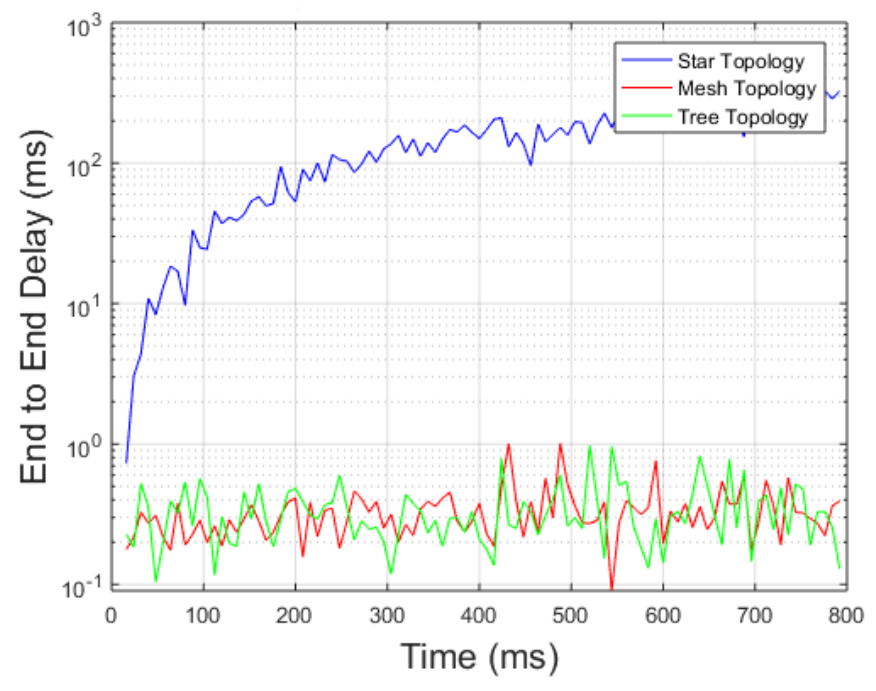

Fig. 7. End to End Delay (mili sec) of the three ZigBee topologies at 868 $\mathrm{MHz}$ transmission band.

The throughput of $868 \mathrm{MHz}$ transmission band show that the star topology has the best performance at this band from Fig. 6 . While the throughput of mesh and tree topologies are much less compared to star topology. From Fig. 7 the worst case of end to end delay in this transmission band and also in all other cases of transmission bands is marked for star topology. The end to end delay of tree and mesh topologies are convergent with slightly less delay for mesh topology.

To summarize the results of Riverbed Modeler simulation for the throughput and end to end delay, the average values of all topologies and transmission band of ZigBee are collected in table IV.
TABLE IV

RESULTS SUMMARY

\begin{tabular}{|c|c|c|c|}
\hline \multicolumn{4}{|c|}{ (1) } \\
\hline & \multicolumn{3}{|c|}{ Topology } \\
\hline & Star & Mesh & Tree \\
\hline $\begin{array}{l}\text { Average Throughput in (Kbps) } \\
\text { for } 2.4 \mathrm{GHz} \text { Band }\end{array}$ & 39.046 & 65.384 & 81.433 \\
\hline $\begin{array}{l}\text { Average Throughput in (Kbps) } \\
\text { for } 915 \mathrm{MHz} \text { Band }\end{array}$ & 29.948 & 23.624 & 21.802 \\
\hline $\begin{array}{l}\text { Average Throughput in (Kbps) } \\
\text { for } 868 \mathrm{MHz} \text { Band }\end{array}$ & 17.052 & 5.287 & 5.135 \\
\hline $\begin{array}{l}\text { Average End to End Delay in } \\
(\mathrm{ms}) \text { for } 2.4 \mathrm{GHz} \text { Band }\end{array}$ & 0.0168 & 0.0259 & 0.0356 \\
\hline $\begin{array}{l}\text { Average End to End Delay in } \\
\text { (ms) for } 915 \mathrm{MHz} \text { Band }\end{array}$ & 90.9564 & 0.4583 & 0.3962 \\
\hline $\begin{array}{l}\text { Average End to End Delay in } \\
(\mathrm{ms}) \text { for } 868 \mathrm{MHz} \text { Band }\end{array}$ & 154.1981 & 0.3327 & 0.3432 \\
\hline
\end{tabular}

For star topology, it can be noticed from table 4. that the End to End delay increases significantly and the throughput decreases with the change of transmission band from $2.4 \mathrm{GHz}$ to lower transmission bands of $915 \mathrm{MHz}$ and $868 \mathrm{MHz}$. The reason for that is at $2.4 \mathrm{GHz}$ transmission band the bit rate is $250 \mathrm{Kbps}$ while the bit rates for $915 \mathrm{MHz}$ and $868 \mathrm{MHz}$ is 40 Kbps and $20 \mathrm{Kbps}$ respectively. Since the communication between end devices is done through coordinator only in this topology, for the same simulation time and same data transmitted, the End to End delay increases and throughput degreases with the decreases of bit rate. Specially the modulation type used for $2.4 \mathrm{GHz}$ transmission band is $\mathrm{O}$ QPSK, while the modulation type used for $915 \mathrm{MHz}$ and 868 $\mathrm{MHz}$ is BPSK.

For mesh and tree topologies, also from table 4. it can be noticed that the End to End delay increases slightly and throughput decreases significantly with the change of transmission band from $2.4 \mathrm{GHz}$ to lower transmission bands of $915 \mathrm{MHz}$ and $868 \mathrm{MHz}$. in the case of mesh and tree topologies the effect of transmission band on End to End delay is little because the communication between end devices is done through multiple routs not only through the coordinator on the opposite of star topology case.

\section{CONCLUSIONS}

The effect of ZigBee network topology on performance is evaluated for throughput and end to end delay for three transmission bands: $2.4 \mathrm{GHz}, 915 \mathrm{MHz}$ and $868 \mathrm{MHz}$. There are three topologies in ZigBee network: star, mesh and tree. For each ZigBee topology, the three transmission bands are evaluated for two important parameters, throughput and end to end delay. In some IoT applications of ZigBee network a low end to end delay is required, while high throughput may be required for other applications. This study shows the performance of possible cases available, so the planner for new ZigBee network can optimize it according to what is needed from the specific IoT application. For table 4. It is found that ZigBee Network that using tree topology at $2.4 \mathrm{GHz}$ achieve 
the highest throughput, while at $915 \mathrm{MHz}$ and $868 \mathrm{MHz}$, the highest throughput can be obtained using star topology. Also from table 4, it can be shown that the minimum end to end delay for $2.4 \mathrm{GHz}$ can be achieved by using star topology, while for $915 \mathrm{MHz}$ the minimum end to end delay is obtained by using tree topology. Finally, for $868 \mathrm{MHz}$, the minimum end to end delay is achieved by using mesh topology. It is found that the end to end delay for star topology at $915 \mathrm{MHz}$ and $868 \mathrm{MHz}$ bands is very high. The results of throughput and end to end delay of mesh and tree topologies show that they are convergent at $915 \mathrm{MHz}$ and $868 \mathrm{MHz}$ bands, especially at 868 $\mathrm{MHz}$ band, with slightly better performance of mesh over tree topology. For $2.4 \mathrm{GHz}$ band, the highest throughput can be obtained by using tree topology, while the minimum end to end delay at this band can be achieved by using star topology. The end to end delay for star topology for lower frequency bands such as $915 \mathrm{MHz}$ and $868 \mathrm{MHz}$ start to increase very much compared to mesh and tree topologies, which is not acceptable for most IoT applications. To summarize conclusions, for IoT applications that are sensitive to delay: all three topologies have acceptable End to End delay with star topology slightly better than mesh and tree topologies at $2.4 \mathrm{GHz}$ transmission band, so the three topologies can be used in this band. While for $915 \mathrm{MHz}$ and $868 \mathrm{MHz}$ transmission bands the delay of star topology is very high and unacceptable, so it's not recommended to use star topology in these transmission bands, instead its recommended to use meh or tree topologies. For IoT applications that requires to transfer large amount of data: its recommended to use mesh or tree topologies at $2.4 \mathrm{GHz}$ transmission bands which provides higher throughput compared to star topology, with tree topology provides the highest throughput at this band. While for $915 \mathrm{MHz}$ and 868 $\mathrm{MHz}$ transmission bands, its recommended to use star topology which provides higher throughput at these bands compared to mesh and tree topologies, especially for $868 \mathrm{MHz}$ transmission band, star topology provides very high throughput compared to mesh and tree topologies.

\section{REFERENCES}

[1] B. R. Stojkoska, A. P. Avramova and P. Chatzimisios, "Application of Wireless Sensor Networks for Indoor Temperature Regulation", International Journal of Distributed Sensor Networks, vol. 2014, Article ID 502419, 2014, Hindawi Publishing Corporation. doi: $10.1155 / 2014 / 502419$

[2] H. Y. Tung, K. F. Tsang, H. C. Tung, V. Rakocevic, K. T. Chui and Y. W. Leung, "A WiFi-ZigBee Building Area Network Design of High Traffics AMI for Smart Grid", Smart Grid and Renewable Energy, vol. 3, pp. 324-333, November 2012. doi: 10.4236/sgre.2012.34043

[3] Y. B. Lin , K. C. Chang, J. C. Chen, P. H. Wang and L. S. Li, "Zigbee Sensor Network For Structural Health Monitoring", 4th International Conference on Earthquake Engineering, Taipei, Taiwan, No. 196, 12-13 October, 2006

[4] M. Rashid and R. Faiz, "ZigBee: Simulation and Invastigation of Star and Mesh Topology by Using Different Transmission Bands", The AIUB Journal of Science and Engineering (AJSE), Vol. 14, No. 1, pp. 115-121, August 2015.

[5] Nimi T. and P. Samundiswary, "Comparative Analysis of ZigBee Network with Tree and Mesh Topology for different range of frequencies", 2nd International Conference on Communication and Electronics Systems (ICCES 2017), Coimbatore, India, No. 113, pp. 560-564, 19-20 October 2017, IEEE. doi: 10.1109/INNOVATIONS.2006.301905

[6] Manpreet and J. Malhotra, "Simulation Analysis of Tree and Mesh Topologies in Zigbee Network", International Journal of Grid and Distributed Computing, Vol. 8, No. 1, pp. 81-92, 2015. doi: 10.1016/j.tust.2017.08.018

[7] P.Mounika, "Performance Analysis Of Wireless Sensor Network Topologies For Zigbee Using Riverbed Modeler", Second Internationa Conference on Inventive Systems and Control (ICISC 2018) Coimbatore, India, pp. 1456-1459, 19-20 January, 2018. doi 10.1109/icisc.2018.8399050

[8] A. Lavric, V. Popa, I. Finis and C. Males, "Performance evaluation of Tree and Mesh ZigBee Network Topologies used in Street Lighting Control Systems", Przegląd Elektrotechniczny, vol. 89, No. 4, pp. 168171, 2013. doi: 10.1016/j.procs.2016.08.041

[9] M. Alnuaimi, K. Shuaib and I. Jawhar, "Performance Evaluation of IEEE 802.15.4 Physical Layer Using MatLab/Simulink", 2006 Innovations in Information Technology, Dubai, United Arab Emirates, 19-21 November 2006, IEEE. doi: 10.1109/innovations.2006.301905

[10] M. A. Moridia, Y. Kawamura, M. Sharifzadeh, E. K. Chanda, M. Wagner and $\mathrm{H}$. Okawa, "Performance analysis of ZigBee network topologies for underground space monitoring and communication systems", Tunnelling and Underground Space Technology, Vol. 71, pp. 201-209, 2018. doi: 10.1016/j.tust.2017.08.018

[11] S. Farahani, ZigBee Wireless Networks and Transceivers, Elsevier Ltd Publication, USA, 2008. doi: 10.1016/b978-0-7506-8393-7.00013-3

[12] M. Ouadou, O. Zytoune, D. Aboutajdine, Y. El Hillali and A. MenhajRivenq, "Improved Cluster-Tree Topology Adapted for Indoor environement in Zigbee Sensor Network", ScienceDirect, Procedia Computer Science, vol. 94, pp. 272-279, 2016. doi: 10.1016/j.procs.2016.08.041

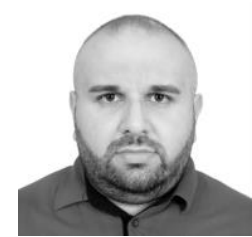

Yehia R. Hamdy was born in Mosul city, Ninawa, Iraq in 1981. He received the B.Sc. degree in Electrical Engineering / Electronics and Communications from University of Mosul, Iraq, in 2005 and M.Sc. degree in Communications Engineering from University of Mosul, Iraq, in 2011. Mr. Yehia is currently a lecturer at the Department of Electrical Engineering, College of Engineering, at University of Mosul. His research interests include: mobile communications, antenna and propagation, positioning and geolocation, telecommunications and optical communications.

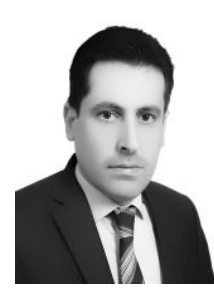

Ahmed I. Alghannam was born in Mosul city, Ninawa, Iraq in 1986. He received the B.Sc. degree in electrical Engineering / Electronics and Communications from University of Mosul, Iraq, in 2008 and M.Sc. degree in Computer Networks Engineering from University of Mosul, Iraq, in 2015.

Mr. Ahmed is currently a lecturer at the Department of Electrical Engineering, College of Engineering, at University of Mosul. His research interests include: computer networks, wireless sensor networks, internet protocols and routing algorithms. 